\section{Intersections}

Canadian Journal of Music

Revue canadienne de musique
Intersections CANADIAN JOURAL OF MUSIO

\title{
Pierre Boulez et le Théâtre de la Cruauté d'Antonin Artaud : De Pelléas à Rituel, in memoriam Bruno Maderna
}

\section{Brice Tissier}

Volume 28, numéro 2, 2008

URI : https://id.erudit.org/iderudit/029954ar

DOI : https://doi.org/10.7202/029954ar

Aller au sommaire du numéro

Éditeur(s)

Canadian University Music Society / Société de musique des universités canadiennes

ISSN

1911-0146 (imprimé)

1918-512X (numérique)

Découvrir la revue

Citer cet article

Tissier, B. (2008). Pierre Boulez et le Théâtre de la Cruauté d'Antonin Artaud : De Pelléas à Rituel, in memoriam Bruno Maderna. Intersections, 28(2), 31-50. https://doi.org/10.7202/029954ar
Résumé de l'article

Dans un texte de 1948, Pierre Boulez exprime son aspiration à investir sa musique d'un souffle rituel comparable à celui de la poétique d'Antonin Artaud. Ces propos soulèvent de nombreuses questions quant à l'influence artaldienne, touchant à la fois les activités de chef d'orchestre de Boulez et la conception de ses oeuvres. Cet article s'attarde d'abord aux considérations de Boulez sur Pelléas et Mélisande dans ses écrits publics, et aux liens qu'il établit entre Debussy et Artaud dans ses écrits privés. Une seconde partie se concentre sur les liens sous-jacents qui relient l'oeuvre d'Artaud et celle de Boulez, plus particulièrement dans Rituel, in memoriam Bruno Maderna.
Tous droits réservés (C) Canadian University Music Society / Société de musique des universités canadiennes, 2009
Ce document est protégé par la loi sur le droit d'auteur. L’utilisation des services d’Érudit (y compris la reproduction) est assujettie à sa politique d'utilisation que vous pouvez consulter en ligne.

https://apropos.erudit.org/fr/usagers/politique-dutilisation/ 


\title{
PIERRE BOULEZ ET LE THÉÂTRE DE LA CRUAUTÉ D'ANTONIN ARTAUD : DE PELLÉAS À RITUEL, IN MEMORIAM BRUNO MADERNA
}

\author{
Brice Tissier
}

Deux traditions se sont rencontrées.

Mais nos pensées cadenassées

N'avaient pas la place qu'il faut,

Expérience à recommencer

Antonin Artaud

\section{INTRODUCTION}

Compositeur, pédagogue, théoricien, chef d'orchestre, Pierre Boulez est un artiste aux activités multiples, mais aussi un auteur particulièrement prolixe. Articles, ouvrages théoriques, entretiens, correspondances publiées. ${ }^{1}$ Boulez traite dans ses écrits bon nombre de sujets : évidemment ses propres œuvres et sa pensée musicale, ses auteurs fétiches, la situation de la musique contemporaine, les institutions françaises... Mais il existe un domaine où Boulez se fait discret, et tout spécialement quant à son œuvre propre : l'esthétique musicale. En 1948, il écrit même :

J'ai enfin une raison personnelle pour donner une place si importante au phénomène rythmique. Je pense que la musique doit être hystérie et envoûtement collectifs, violemment actuels - suivant la direction d'Antonin Artaud et non pas dans le sens d'une simple reconstitution ethnographique à l'image de civilisations plus ou moins éloignées de nous. Mais là encore, j'ai horreur de traiter verbalement ce qu'on nomme avec complaisance le problème d'esthétique. Aussi ne prolongerai-je pas davantage cet article; je préfère retourner à mon papier réglé. (Boulez 1948, p. 262)

Boulez élude le problème esthétique mais cite un nom, celui de l'inventeur du Théâtre de la cruauté. Or, surprenante constatation à la lecture de livres et articles : très peu de références au poète de Rodez. Alors pourquoi cette affirmation passagère ? Et pour aller plus loin : Boulez aurait-t-il envisagé cette esthétique du Théâtre de la cruauté pour ses interprétations du répertoire? Pourquoi

1 L'étendue de ce corpus immensurabilis apparaît dans la récente édition intégrale des écrits par Jean-Jacques Nattiez. Trois tomes sont parus - Points de Repère I : Imaginer, Regards sur autrui, Leçons de musique; un quatrième est prévu. 
aucune composition sur des textes d'Artaud dans le corpus officiel de ses propres œuvres? Il y a des réponses à ces questions. Il existe chez Boulez une réelle dichotomie des écrits, selon deux catégories : d'une part ceux destinés à la publication (articles, textes théoriques, entretiens et conférences), et d'autre part les écrits privés (correspondances, esquisses préparatoires aux compositions musicales, etc. $)^{2}$. Le cas 'Antonin Artaud' reflète cette dichotomie. Si dans la première catégorie Boulez esquive le sujet (comme il admet lui-même éviter les questions esthétiques), il n'en va pas de même dans la seconde. La conception boulézienne de Pelléas et Mélisande de Debussy dans les écrits édités suit cette logique, comme nous allons le voir en la comparant avec les rapprochements Debussy/Artaud dans ses écrits privés. Dans une seconde partie nous parlerons des liens existant entre l'œuvre d'Artaud et celle de Boulez.

\section{De Pelléas et Mélisande au Théâtre de la Cruauté}

\section{Pelléas dans les écrits de Boulez}

Auteur d'une notice biographique de Debussy en 1958 pour l'encyclopédie Fasquelle (Boulez 1985, p. 210-217), conducteur de son répertoire orchestral dès les années 1950, il était inévitable de voir Boulez s’intéresser à l'unique opéra achevé de l'auteur de La Mer. "Miroirs pour Pelléas et Mélisande » (1969) (Boulez 1985a, p. 433-446) est un article théorique et rédigé. ${ }^{3}$ À ce titre, ce texte ne constitue pas qu'une simple vision d'interprète : plutôt un miroir de ses réflexions préalables à son approche concrète de l'œuvre. Globalement les « Miroirs » s'inscrivent dans la continuité de la notice de 1958. L'auteur ne ménageait guère alors ses critiques contre Pelléas et surtout le livret de Maeterlinck. Mais si Boulez ne manque pas de revenir sur la question, "Miroirs » tend à traiter en finalité de l'œuvre musicale. Pour lui Pelléas reste une ouvre incomprise n'ayant pas connu, dans la vie de Debussy comme dans l'histoire globale de l'opéra, la vaste popularité à laquelle elle devrait prétendre. Est-ce en raison du manque de chant? de grandes expansions orchestrales? du caractère désuet du théâtre de Maeterlinck ? ou encore du malentendu quant à une «spécificité nationale " ? Si Pelléas tient une place importante dans la production debussyste (première œuvre scénique achevée, utilisation de thèmes poétiques récurrents comme la chevelure ou la mer, affirmation de la personnalité et des caractéristiques esthétiques du compositeur), il n'en va pas de même dans l'histoire du genre. Pour Boulez, la relative indifférence du public serait grandement imputable à l'écriture théâtrale de Maeterlinck, statique et incolore, à son influence sur la musique, et à la frilosité des amateurs d'opéra : «Rien n’a

2 Dans son ouvrage La transcription dans Boulez et Murail, Éric Humbertclaude (1999) avait déjà mis en évidence cette dichotomie à travers l'étude des partitions de Boulez sous leurs deux formes : la partition composée, présentant visuellement les arcanes de la pensée boulézienne, et la partition définitive, où ces arcanes sont désormais dérobées. Humbertclaude prend notamment en exemple les partitions comparées de la Notation II pour orchestre.

3 Il s'inscrit dans le cadre de la première production boulézienne de Pelléas, du $1^{\text {er }}$ au 31 décembre 1969, avec une mise en scène de Václav Kaslík et Josef Svoboda; édité pour la circonstance dans le programme de Covent Garden, puis repris l'année suivante pour le livret d'accompagnement de l'enregistrement. 
beaucoup changé, depuis lors, en ce qui concerne les goûts quotidiens, dans ce domaine par excellence du conservatisme le plus militant. » (Boulez 1985a, p. 421) Et pourtant rien ne semble manquer au drame : « des ingrédients et des épices : l'amour, la jalousie, la violence, la malédiction, le meurtre. " (Ibid.) Pour Boulez, le véritable problème de Pelléas réside dans une dimension également prégnante pour Tristan et Isolde : la relation entre symbolisme et réalisme. Car contrairement à leurs homologues wagnériens, les amants maeterlinckiens n'ont jamais acquis le statut de symbole : si les deux héros sont intemporels, ils restent ancrés dans un drame somme toute très quotidien auquel manque cette dimension symbolique. Et Boulez de citer l'exemple de la chevelure : au rituel cruciforme de l'acte III qui a besoin « du réalisme scénique pour se manifester dans toute son énergique cruauté » (Boulez 1985a, p. 422) s'oppose l'irréalisme absolu de la perruque de l'acte II, impossible instrument de la future torture. Or selon Boulez, la conception théâtrale de Debussy se fonde sur le flux et le reflux entre «le réalisme le plus implacable et l'onirisme le plus impalpable» (Ibid.). Un concept fondamental chez Edgar Poe, ce qui vouait fatalement à l'échec le projet debussyste de la Chute de la maison Usher, second Pelléas en puissance. Latmosphère de Pelléas se doit d'être sombre, pesante et sans soleil. Elle se double d'une caractérisation des protagonistes ayant « [...] de quoi décourager, ou enrager, les plus intrépides amateurs de rêves » (Ibid.) : une Mélisande planante, un Pelléas en page distingué, un Golaud "fier-à-bras ", un Arkel naïf (dont les répliques se révèlent être davantage des aspirations que de véritables prophéties), une Geneviève résignée... Selon Boulez, Golaud, seul personnage actif de l'opéra, en est le personnage central. Cette interférence entre réalisme et symbolisme constitue aussi la caractéristique musicale principale de Pelléas. Elle correspondrait même à l'une des données les plus élémentaires du genre opératique depuis Monteverdi : la dualité action/commentaire. Cette dualité, très marquée par le passé, assurait une plus grande efficacité du cadre formel, avec par exemple la disjonction récitatif/aria, à laquelle répondaient par ailleurs d'autres dualités (comme les fonctions dramatiques information/ expression et solo/ensembles). Chez Wagner, cette dissociation s'efface au profit de l'uniformité de la mélodie continue et le déploiement de grandes plages sonores - nonobstant les quelques chœurs des Meistersinger. Pour Boulez c'est, chez Debussy, l'exact contraire : la préservation de cette dichotomie, ramenée au niveau microformel : «Il nous donne un tissu extrêmement serré d'action et de réflexion. Il faut, dès lors, avoir l'œil aigu et l'oreille fine pour percevoir ces différences, ces modulations : elles passent rapidement; à peine si, parfois, le changement stylistique - vocal ou instrumental - est amorcé, esquissé. » (Boulez 1985a, p. 425) Boulez trouve ici les origines de l'incompréhension de l'œuvre : à force de ne voir en Pelléas qu'un récitatif continu, en réaction contre la mélodie infinie de Wagner, on a escamoté sa véritable nouveauté. Qu’advient-il dans ce cas de la continuité musicale ? Celle-ci est évidemment assurée par les leitmotivs, qu'il faut une fois encore concevoir différemment de la perspective wagnérienne : l'influence du compositeur allemand ne se traduit ici que par la caractérisation thématique des personnages, sans mutations ou développements structurels. Il est une évidence : Boulez ne cesse de comparer Debussy 
à Wagner, et pas seulement dans l'intention de les dissocier. Il existe selon lui une réelle influence de Parsifal, à travers notamment l'instrumentation - dans les intermèdes composés tardivement pour meubler les changements de décors, mais aussi dans la caractérisation des personnages. Il serait légitime de rapprocher la figure rythmique de Golaud avec le thème de Parsifal; de même pour les personnalités musicales d'Arkel et Gurnemanz. Loin de constituer un manifeste antiwagnérien agressif, comme on le présente souvent, Pelléas trouve en Wagner l'une de ses sources principales.

Pour conclure, il serait nécessaire d'évoquer brièvement aussi les quelques remarques «techniques » de Boulez quant à l'interprétation matérielle de Pelléas, ce qu'il appelle la «libération de Pelléas». Encore une fois réactionnaire vis-à-vis des traditions institutionnelles, ce dernier prône un réel retour à la partition, tant musical (en évitant d'étouffer l'orchestre au profit des voix et en assurant une meilleure gestion des contrastes sonores), que dans les choix préalables de la distribution, avec par exemple l'attribution du rôle d'Yniold à un enfant, et celui de Pelléas à un véritable ténor.

Boulez depuis est resté fidèlement attaché à ces jugements qui, conformément à son style, ne laissent guère de place à la discussion. L'interview qu'il accorde à James R. Briscoe trente années après les « Miroirs » le démontre : ses idées sur la forme et la conception globale restent immuables et reprennent, dans les grandes lignes, celles des « Miroirs ».4 En revanche, cette interview aborde deux questions nouvelles : son évolution propre dans l'interprétation debussyste - et de Pelléas en particulier, chose impossible en 1969 - et l'histoire de l'interprétation de l'opéra depuis 1902. Cela dit, on ne saurait attendre une autocritique de la part de Boulez, ni même un point de vue rétrospectif personnel... Boulez ne se réécoute pas, et de manière générale se refuse à la rétrospection, symptôme d'une faiblesse intellectuelle ou civilisationnelle. C'est probablement d'ailleurs pour cette raison qu'il accepte de réenregistrer «son » répertoire. Mais au-delà de la trace discographique, Boulez ne conteste pas l'évolution qu'accomplit la seconde production de 1992. Si celle-ci ne se conçoit pas sur le fond (la partition et son interprétation), elle se pose au niveau de la forme, à travers ce que Boulez nomme le « regard circonstanciel du chef d'orchestre ». Malgré le travail musical exemplaire et méticuleux de préparation de la partition 5 , Boulez exprime quelques regrets quant à la présentation terminale de la première production, sans réellement en préciser l'objet. Sur le plan musical, il admet aussi une évolution stylistique de son approche de l'opéra. S’il

4 Précisons que l'influence des « Miroirs » est flagrante dans les questions de James Briscoe, qui s'en est très probablement inspiré. D’où des réponses de Boulez dans cette même proximité. On peut également supposer que, l'exercice de l'interview ne permettant pas une préparation préalable ni une réflexion poussée, Boulez dans l'instant n'ait pas développé plus avant dans ses réponses ses conceptions sur Pelléas qui auraient pu évoluer durant ces trente années. On ignore par exemple s'il publia, comme en 1969, un texte dans le programme de l'opéra.

5 On sait les relations parfois tendues entre Boulez et les institutions orchestrales, quant à l'organisation des répétitions à cette époque. Faisant fi des conventions, le compositeur n'accepte alors de diriger une production qu'à la condition sine qua non de bénéficier d'un plan de travail adapté, suffisant et rigoureux (par exemple 17 répétitions tutti pour Wozzeck à l'Opéra de Paris en 1973). Nul doute que la situation était équivalente à Covent Garden. 
ne revient pas sur le fond, Boulez reconnaît une approche probablement plus dramatique et plus théâtrale qu'en 1969, ainsi qu'une propension nouvelle à accentuer davantage les contrastes musicaux.

Le texte aborde également l'histoire de l'interprétation de Pelléas depuis sa création, discutant l'approche des chefs d'orchestre de la première moitié du $\mathrm{XX}^{\mathrm{e}}$ siècle, et soulevant implicitement le problème de l'authenticité. Boulez, il faut bien le dire, ne cache pas son scepticisme quant au concept de représentation authentique : les compositeurs ne peuvent imaginer la part théâtrale de leurs œuvres qu'en fonction des canons stylistiques de leur époque. Mais toute tentative de préserver cette dimension dans la période actuelle serait non seulement ridicule, mais aussi obsolète. ${ }^{6}$ Pour illustrer ce propos, signalons par exemple le rejet boulézien de toute indication scénique ou logistique (didascalies en tête).7 Représenter un opéra aujourd'hui - qu'il s'agisse de Debussy, Berg, Schoenberg ou Wagner - nécessite de trouver une solution que le compositeur aurait trouvée dans notre propre environnement. Interrogé enfin sur ses prédécesseurs (dont, comme le souligne James Briscoe, des enregistrements préservent encore aujourd'hui des interprétations proches de la date de création) le compositeur reste encore une fois sceptique, fidèle à la formule darmstadtienne : du passé faisons table rase. À l'image de ses propres enregistrements Boulez refuse l'épreuve du temps, et particulièrement le jugement sur l'évolution dans le temps. (Boulez et Briscoe 1999, p. 182-183) Il faudrait même se méfier de la proximité temporelle entre artistes et interprètes, et à fortiori des œuvres interprétées par les compositeurs eux-mêmes. Comme Boulez le souligne, Stravinsky restera hélas un bien piètre conducteur de ses propres œuvres orchestrales, et Bartók un pianiste contestable, dans le jeu de ses propres compositions pour clavier. On le comprend : Boulez élude encore ces questions.

À la lecture de ces deux textes, et des « Miroirs » en particulier, nous pouvons faire trois remarques générales. La première concerne le point de vue d'un Boulez, qui ne saurait finalement faire preuve d'objectivité ! Si « Miroirs » cherche à dépoussiérer une partition institutionnellement chargée, on ne peut s'empêcher de voir, en filigrane, un positionnement géographico-culturel personnel ${ }^{8}$, ainsi que les échos de ses critiques anciennes dirigées contre les institutions et les mentalités françaises en général, notamment sur la question de Debussy. Il s'en prend aux « thuriféraires locaux ayant asphyxié leur idole avec de l'encens de qualité douteuse ! »(Boulez 1985b, p. 428)9, rappelant au passage que Debussy lui-même éprouvait une répulsion pour l'opéra de son temps, et composait vers l'avenir, le matin en regardant le soleil se lever ! Le deuxième élément important de ces deux textes concerne le parallèle que Boulez dresse entre le Debussy de Pelléas et le Wagner de Tristan et Parsifal. Outre d'évidentes

6 On retiendra à ce sujet sa phrase particulièrement claire au sujet de la Cerisaie, présentée par la compagnie Stanislavski originelle : «Ce n'était pas une pièce de musée, c'était une pièce de mausolée !! ».

7 Il rejette même le concept de mise en scène pour le Château de Barbe-Bleue en 1998 à Aix-enProvence, au profit d'une chorégraphie de Pina Bauch.

8 Depuis 1966, Boulez vit à Baden-Baden, après avoir claqué la porte du monde musical français ayant choisi, avec Malraux et Landowski, une politique conservatiste.

9 Voir surtout l'article « La corruption dans les encensoirs », 1956, dans Boulez 1985 b. 
similitudes entre ces trois opéras, Boulez défend clairement l'assimilation d'un héritage wagnérien dans l'écriture debussyste, et il n'est pas impossible qu'il lie l'échec relatif de son projet originel de production de Pelléas à Covent Garden à cette question. ${ }^{10}$ Cependant, bien que Boulez reconnaisse l'imperfection de la mise en scène de cette première production, il ne renvoie pas au côté wagnérien du problème. ${ }^{11}$ Finalement, la donnée probablement la plus essentielle du commentaire de ces deux textes réside dans le mystère enveloppant la conception esthétique profonde de Boulez, c'est-à-dire les sources ou influences souterraines qui motivent sa vision de l'œuvre. Au-delà des critiques, il évoque certes une atmosphère sombre, pesante, sans soleil telle que décrite dans les didascalies, mais pour Boulez, il est fort probable que cette interprétation ne soit pas uniquement scripturaire - on sait son désintérêt perdurant pour les indications scéniques, didascalies ou autres annotations sclérosantes relevant d'un Zeitgeist néfaste à la pérennisation des œuvres.

C'est là qu'intervient une source d'inspiration externe quant à la conception du drame de Debussy, conception que Boulez ne fait qu'évoquer subrepticement dans « Miroirs » (Boulez 1985, p. 428) : le Théâtre de la cruauté d'Antonin Artaud, qui selon lui constitue la meilleure approche possible de la substantifique moelle de l'œuvre. Sa correspondance ainsi que ses rapports avec André Schaeffner font clairement état de cette influence, et cautionnent jusqu'à l'existence même du texte "Miroirs "; mais cette dimension reste cependant cruellement en retrait dans l'article.

Boulez fait la connaissance d'André Schaeffner ${ }^{12}$ dans les années 1950, et entretient depuis lors une riche correspondance avec lui, aujourd'hui publiée (Schaeffner et Boulez 1998). Au sein de cette correspondance, nous trouvons des réponses à notre affirmation artaldo-boulézienne initiale.

\section{Boulez-Schaeffner : vers une approche artaldienne de Pelléas}

\section{La correspondance Boulez-Schaeffner}

C'est en 1961 que la question s'ouvre dans les échanges entre le compositeur et l'ethnologue, même si l'on peut supposer que l'interprétation de Pelléas est un sujet d'intérêt pour Boulez depuis longtemps déjà. À l'époque, Boulez travaille à une nouvelle interprétation du Pierrot lunaire, qu'il souhaite réformer. Il s'adresse alors à Schaeffner, auteur de plusieurs articles en accord avec ses propres idées ${ }^{13}$. Schaeffner écrit :

10 La production de 1969 à Covent Garden était en effet prévue à l'origine avec une mise en scène de Wieland Wagner, donc un lien affirmé avec le compositeur allemand que Boulez commençait à diriger alors à Bayreuth.

11 On ne saurait aussi expliquer la seconde production de Pelléas par le seul mécontentement du chef vis-à-vis de la production de 1969. À cette époque Boulez entame un contrat d'exclusivité avec Deutsche Grammophon; il réenregistre donc tout son répertoire (Debussy, Ravel, Stravinsky, les trois Viennois, etc.), gravé chez Sony dans les années 60.

12 Ethnologue, historien et critique musical et littéraire, il est le créateur du département d'ethnologie musicale du Musée d'ethnographie du Trocadéro (1929), puis du Musée de l'homme (19371964).

13 Notamment dans Variations Schoenberg, In Schaeffner, 1980. 
Et si vous parlez du « décrassage » du Pierrot, tout cet été, et encore à mes moments soi-disant perdus, je me suis mis et m'emploie à un autre décrassage, celui de Pelléas. Mais, à l'inverse de ce que vous faites pour Pierrot, je remets de la terreur dans Pelléas et même quelque peu du Théâtre de la cruauté selon Artaud! (Pereira de Tugny 1998, p. 42) ${ }^{14}$

La réponse de Boulez quelques jours plus tard semble être la première trace scripturaire de sa réflexion :

Merci pour Pelléas. Les deux scènes entre Golaud et Mélisande sont horribles ! Surtout cette torture du $5^{\mathrm{e}}$ acte; cet horrible monsieur qui veut tout savoir. De toute façon, c'est l'œuvre entière de Debussy qu'on dirige, ou qu'on joue trop mou. Il y a des pointes de brutalité ! C'était un musicien nerveux, et pas ce voluptueux lymphatique qu'on en a fait comme caricature. (Ibid., p. 50 $)^{15}$

Boulez dirigera Pelléas à Londres du $1^{\mathrm{er}}$ au 31 décembre 1969, avec un certain succès, remarqué notamment outre-Manche ${ }^{16}$. L'enregistrement sera effectué dans la foulée. ${ }^{17}$ Trois textes l'accompagnent : une documentation de F. Aprahamian sur la création avec données biographiques et iconographie, le fameux texte "Miroirs pour Pelléas et Mélisande » repris du programme de Covent Garden, et un troisième texte demandé par Boulez à Schaeffner :

J'aimerais vous demander [...] si vous vouliez écrire un texte - ou une variation du texte existant - sur Debussy et le Théâtre de la cruauté en replaçant Pelléas au milieu de ses efforts inaboutis de théâtre, spécialement $L a$ chute de la maison Usher. J'aimerais pouvoir produire votre texte à l'appui de la musique; toutes les relations profondes entre Usher et Pelléas sont à mettre en évidence, afin qu'on voie bien que Debussy n'était pas ce débile molasson qu'on présente comme le fin du fin de la musique française entre les marrons glacés et Chanel $\mathrm{n}_{4}^{\circ}$ (ou 5 ou 10 ?). Et surtout comme vous l'avez déjà fait, il faudrait replacer Pelléas à l'intérieur des fantasmes et obsessions de Debussy concernant le théâtre... (Ibid., p. 108-109) ${ }^{18}$

Ce texte ${ }^{19}$, Schaeffner en accepte alors la refonte, mais avec quelques modifications :

Il va de soi que l'essai que je vous destine sera tout de même un peu différent, d'abord plus court, ensuite insistant sur d'autres points, sinon sur un point. J'ai eu l'occasion de reprendre la presse de 1902, lors de la création

14 Lettre du 22 novembre 1961. Artaud à l'époque exerce une certaine influence sur plusieurs metteurs en scène dont Roger Blin, Jean-Louis Barrault et Julien Beck.

15 Post-scriptum manuscrit à la lettre postée le 24 novembre 1961.

16 On notera un excellent compte-rendu de Jacques Lonchampt (ex directeur de l'opéra de Paris, qui avait notamment soutenu Boulez lors du projet de production de Wozzeck en 1963) daté du 4 décembre 1969. Impression relayée par Georges Auric à André Schaeffner peu après à l'Institut de France. (Pereira de Tugny 1998, p. 111). pour CBS (M9 30119).

17 Pour CBS (M9 30119).

18 Lettre de Boulez à Schaeffner, postée à Londres le 3 décembre 1969.

19 «Debussy et le théâtre ", qui deviendra " Théâtre de la peur ou théâtre de la cruauté ?, préface à Edgar Poe et Debussy ", repris dans Schaeffner 1998. 
de Pelléas, et ai été frappé du peu qu'on en avait tiré. Que ce soit Vincent d'Indy ou Julien Benda (quel âge avait-il ?) ou quelques autres (Octave Mirbeau, Zola, Bruneau), ils ont parfaitement vu le côté "théâtre de la Cruauté » de la partition de Debussy. Je suis sûr de trouver encore d'autres témoignages de pareille lucidité. J’ai également relevé, mais ceci dix ans plus tard, une appréciation d'un ami de Gide (Henri Ghéon) sur la façon très ferme dont d'Indy dirigeait les Nocturnes - opinion confirmée par un autre critique qui assista à cette exécution des Nocturnes...au Théâtre Marigny (déjà !). Tout en étant sensibles au « charme " de Debussy, certains avaient vu plus loin. (Pereira de Tugny 1998, p. 114) ${ }^{20}$

Cette refonte fera l'objet de nombreuses tractations entre Boulez et Schaeffner ${ }^{21}$; le compositeur semblait surtout avoir été marqué par la dernière remarque de son ami concernant les écrivains contemporains de Debussy. Il le signifie dans une lettre ultérieure :

À vrai dire oui, j'avais espéré une note plus longue et plus détaillée sur les rapports de Debussy avec le théâtre en général. Mais je vous comprends fort bien pour l'avoir expérimenté moi-même. Il est lassant de remettre ses pieds dans des empreintes déjà foulées... C'est de plus très intéressant de voir les rapports de Debussy avec ces écrivains fin de siècle qui n'ont jamais trouvé leur place et qui sont restés, au sens littéral, des ex/centriques. Je suis content que vous ayez aimé l'enregistrement. Il me semble en effet que si l'œuvre a été quelque peu tirée à moi, c'est par l'intérêt porté à Golaud. C'est le personnage que je trouve le plus complexe et le plus attirant. Je crois même que par antiphrase, au lieu d'appeler l'opéra Pelléas et Mélisande, on pourrait simplement l'appeler Golaud... ${ }^{22}$

Précision importante : il ne saurait être question, tant de la part de Schaeffner que de Boulez, d'envisager Debussy dans le cadre strict, théorisé du Théâtre de la cruauté artaldien. Rappelons d'ailleurs que le texte considéré comme le «manifeste » officiel du genre par l'auteur d'Héliogabale date de $1932^{23}$, quatorze années après la mort de Debussy. D’où ce rapport, et surtout cet intérêt exprimé ici par nos deux musicologues pour les "écrivains fin de siècle ", de préférence «ex/centriques ». Le texte de Schaeffner "Théâtre de la peur ou de la cruauté ? " s'inscrit dans cette perspective. Nous devrions donc y comprendre comment ce dernier envisage les liens entre Debussy et le théâtre artaldien. Pour André Schaeffner l'œuvre de Debussy toute entière se doit d'être repensée par le biais de ses lectures et ses influences littéraires. C'est pourquoi ce principal article - mais aussi d'autres plus secondaires comme " Le théâtre imaginaire de Debussy » ou encore "Segalen et Debussy » - ne traitent que la dimension textuelle (influences, lectures et livrets) : peu, voire pas de remarques concernant la musique elle-même. En ce sens, on ne saurait penser unilatéralement les considérations de Boulez et de Schaeffner concernant Pelléas.

20 Lettre de Schaeffner à Boulez du 30 décembre 1969.

21 Notamment dans une lettre de Boulez à Schaeffner du 27 février 1970.

22 Lettre de Boulez à Schaeffner du $1^{\text {er }}$ décembre 1970, postée de New York.

23 Artaud écrira deux manifestes du Théâtre de la cruauté dans la Nouvelle Revue Française, respectivement en octobre 1931 et octobre 1932. 


\section{Les écrits de Schaeffner: Debussy d'Edgar Allen Poe à Antonin Artaud}

Pour Schaeffner, il y a deux hypothèses principales : l'influence centrale des œuvres d'Edgar Allen Poe sur Debussy ${ }^{24}$, puis la question de la dramaturgie dans le contexte biographico-culturel du compositeur de Pelléas, c'est-à-dire une transition d'un Théâtre de la peur (Poe) vers un Théâtre de la cruauté (Artaud). Schaeffner soutient l'idée d'une certaine unité thématique pour l'ensemble des projets - aboutis ou non - de Debussy. Une unité fondée sur les deux concepts de "peur » et de "progression de l'angoisse", constituant les fondements du récit de La chute de la maison d'Usher : «La peur » («Fear») - seul mot de tout le conte à être imprimé en lettres capitales - y est associée aussi à la « femme instrument » (Lady Madeline), voire même à "l'instrument-femme » : des thèmes qui - toujours selon Schaeffner - appartiennent à Debussy. Ce dernier ne cesse de les reprendre, notamment dans son projet de mise en musique du conte de Poe lui-même : «Si je puis réussir, comme je le veux, cette progression dans l'angoisse que doit être La chute de la maison Usher, je crois que j'aurai bien servi la musique... » (Schaeffner 1998, p. 377)25 On le sait, il y faillira. Mais Schaeffner voit dans Pelléas, seize années auparavant, un premier degré de la réalisation de ce Théâtre de l'angoisse et de la terreur. Citant le compositeur : «Et la scène des souterrains fut faite, pleine de terreur sournoise, et mystérieuse à donner le vertige aux âmes les mieux trempées. » (Ibid.) ${ }^{26}$ Une scène des souterrains qui n'est pas sans rappeler la tragédie de Lady Madeline enterrée vivante par son propre frère Roderick Usher! (Sans compter la dualité frappante des deux scènes : une ressemblance entre le mystérieux Usher demandant au naïf Philipp Winhrop de descendre avec lui le cercueil de sa sœur, et le sombre Golaud, menant Pelléas dans les ténèbres...) De même, Schaeffner rapproche la psychologie des personnages de Pelléas et d'Usher, par l'entremise de Debussy lui-même :

Les retouches d'ordre psychologique sont assez nombreuses, nous ne saurions y voir des habiletés de librettiste. Lady Madeline, que Poe dit jumelle de Roderick, est beaucoup rajeunie, et par là rapprochée de Mélisande. Apparition muette dans le conte, elle chante dans le drame de Debussy, mais c'est son frère qui lui fait chanter " des musiques à damner les anges" et la mine aussi peu à peu : «Il ne sent pas que c'est son âme à elle, qui s'en va avec le chant. » (Ibid., p. 378)

Pour l'ethnologue, l'angoisse est ici liée au chant et au concept de " progression ». La " progression vers l'angoisse » plus particulièrement est immanente chez Debussy, on la trouve par exemple dans certaines scènes de Pelléas, le deuxième mouvement de $\mathrm{La} \mathrm{Mer}$ ou encore Jeux, qui sont traversés en entier d'un courant sans cesse interrompu et autant de fois repris, à une intensité de haut voltage, pour se conformer aux exigences de la dramaturgie. L'autre point important du texte concerne une donnée selon lui fondamentale de la drama-

24 Nous laisserons ici de coté les « ex/centriques » (dixit Boulez) dont traite également Schaeffner : Suarès, Bloy, Cros...

25 Lettre du 8 juillet 1910 (Debussy 1927, p. 85).

26 Lettre du 28 août 1894 (Denis 1932, p. 29). 
turgie debussyste : l'influence des musiques et des spectacles d'Extrême-Orient et de Java que le compositeur avait découverts lors des Expositions universelles de 1889 et 1900 . On ne saurait négliger cette perspective, explicitée par Monsieur Croche lorsqu'il décrit le théâtre annamite en 1913. Une description que Schaeffner envisage également comme un mouvement de réaction de Debussy suite au désenchantement wagnérien de ses deux voyages à Bayreuth. L'auteur de Pelléas discerne dans ce théâtre annamite un embryon de drame lyrique « [...] où se reconnaît la formule tétralogique; il y a plus de Dieux, et moins de décors... Une petite clarinette orageuse conduit l'émotion; un Tam-tam organise la terreur... et c'est tout ! (Ibid., p. 389) ${ }^{27}$ L'expression organiser la terreur (dans un texte rédigé l'année de la création du Sacre du printemps rappelonsle $)^{28}$, retient évidemment l'attention de l'ethnologue, mais aussi la dimension extra-culturelle chère à Antonin Artaud, auteur de l'une des principales descriptions des spectacles de Bali en 1931:

Selon Artaud, les artistes balinais usent de tous les moyens susceptibles de produire l'hallucination et la peur; leur orchestre de bronze et de peau organise, lui aussi, la terreur. Les spectacles auxquels Debussy assista en 1889 confondaient quelque peu les deux théâtres. D’après les articles de journaux, l'horreur n'y était pas ménagée; un accouchement, entre autres, trop bien mimé sur scène, causa scandale. (Ibid.)

Ainsi le passage d'un Théâtre de la peur à un Théâtre de la cruauté chez Debussy se ferait-il par le biais de l'ethnomusicologie. Bien sûr nous pourrions reprocher à l'ethnologue de prêcher pour sa paroisse, en ouvrant le style de Debussy sur l'ethnomusicologie sans donner la moindre explication ni même une possible application concrète dans la partition musicale. L'ethnomusicologie constitue cependant, pour Schaeffner, l'une des raisons d'être de la présence de terreur, dans un cadre dramaturgique. Il n'est pas sans rappeler du reste l'utilisation, au début du troisième mouvement de La mer, d'un certain tam-tam, lequel «participait déjà de cette musique d'épouvante dont Edgar Poe n'est pas seul à lui avoir suggéré l'idée. » (Ibid.) Y aurait-il enfin, chez Debussy, filiation directe ainsi entre Théâtre de la peur de Poe et Théâtre de la cruauté esquissé dans les rituels d'Extrême-Orient ? Schaeffner se contente de préciser qu'avant l'Exposition universelle de 1889, nous n'avons connaissance d'aucun projet du compositeur lié aux Histoires extraordinaires.

\section{Pelléas par Boulez: de Schaeffner à l'opéra Golaud}

Dramaturgiquement, la lecture bouézienne de Pelléas est corrélée aux textes d'André Schaeffner. Ainsi que l'écrit Laurent Feneyrou, « il souhaite dévoiler, dans la partition, la violence, la brutalité et la terreur sournoise du drame debussyste. » (Feneyrou 2005, sl.) Dans ses lettres, et bien entendu dans « Miroirs ", Boulez veut réagir contre "l'imagerie lénifiante et pudibonde qui retire à l'œuvre sa substance, ses tensions, ses flux et reflux entre réalisme d'un

27 Monsieur Croche, in S.I.M., 15 février 1913, p. 48.

28 Lui-même avait entendu le Sacre en 1914, ce qui encouragera sa carrière d'ethnologue. 
drame quotidien et l'onirisme de ses thèmes » (Ibid.). Edgar Allen Poe est donc cité fraternellement dans "Miroirs ». Sans revenir sur le contenu de ce texte, on peut probablement expliquer en partie par la lecture des idées de Schaeffner l'attention que porte Boulez aux questions de contraste, de gestion de l'orchestre, et à la valorisation de la puissance et de la violence de l'œuvre. Autrement dit, une conception de l'orchestre comme " apport » et non " support ». Par ailleurs, Boulez adopte les références de Schaeffner à l'Exposition universelle de 1889, aux danses, aux musiques et aux théâtres d'Orient, lesquelles doivent nous mener à " un examen de l'élargissement considérable de l'horizon familier de Debussy, à ce que Boulez nommait dès les années 1950 la 'rupture du cercle d'Occident' " (Ibid.). ${ }^{29}$ Toujours Feneyrou : « Non un usage folkloriste, non un exotisme à bon marché destiné à combler de pittoresque des nostalgies colonialistes, mais la recherche d'une tradition autrement, mais tout aussi puissamment codifiée et d'une poétique imposant son influence 'corrosive' » (Ibid.). Au-delà des convergences avec la pensée de Schaeffner, le fameux recentrement opéré par Boulez sur le personnage Golaud illustre cependant un lien entre Artaud et Pelléas, qui échappe au texte de l'ethnomusicologue. Ciblant l'œuvre sur l'évolution psychologique de Golaud (un être successivement robuste même lorsqu'il est perdu, puis anxieux, dément, en " horrible monsieur qui veut tout savoir ", jusqu'à l'ultime torture qu'il inflige à Mélisande mourante) et reléguant au second plan l'histoire d'amour de Pelléas et Mélisande, Boulez envisage - volontairement ou non - cet opéra dans la lignée des drames artaldiens tels Héliogabale ou Van Gogh. Dans son essai Van Gogh ou le suicidé de la sociétée ${ }^{30}$, Artaud souhaite réhabiliter le peintre des Tournesols, en présentant un plaidoyer contre la folie. De son point de vue, cette dernière ne doit pas être considérée comme un dérèglement psychique, mental ou moral, mais au contraire comme une forme de vision extra-naturelle, surréaliste, élevant l'être à un mode de pensée et d'appréhension du réel supérieur. Pour comprendre Van Gogh, Artaud pense nécessaire de s'extraire du point de vue global de notre société, pour se hisser au niveau de la pensée supérieure de l'artiste. Artaud critique le refus de notre société d'embrasser la pensée supérieure, qu'elle met à l'écart, en faisant interner Vincent Van Gogh et en bridant, par la recherche de l'uniformisation, sa pensée créatrice. Il va de soi qu'à travers cet essai, Artaud envisage parallèlement sa propre situation, sortant alors lui-même de plusieurs années d'internement et de traitements par électrochocs. Selon cette perspective, Golaud serait un héros artaldien, s'élevant au dessus du réel d'un drame amoureux traditionnel, dont il est, tant de son point de vue et - ce qui est particulier ici - du point de vue de la morale elle-même, critique de droit. L'autre perspective, également attribuable à un Golaud central, concernerait le second aspect récurrent des personnages artaldiens : la recherche de héros négatifs. Artaud se plait à traiter et décrire des mondes de vice et de crime, vus dans la perspective temporelle de la déchéance, de la destruction graduelle, et donc la

29 Voir aussi « la corruption dans les encensoirs », 1956, dans Boulez 1985b, p. 157.

30 Présenté tout d'abord sous la forme d'une conférence, Van Gogh, le suicidé de la société sera publié en 1947, quelques mois seulement avant la mort d'Artaud. 
progression de l'angoisse. Son Héliogabale ${ }^{31}$, empereur dépravé d'une Rome décadente et autodestructrice au troisième siècle, poursuivra ses lubies jusqu'à l'excès, jusqu'au point de non-retour et son assassinat. Le Prince Golaud, fils de roi, s'inscrit d'une certaine manière dans ce mode de déchéance ${ }^{32}$. Boulez aurait-il envisagé Golaud comme un personnage artaldien ? Encore une fois aucune source ne vient le confirmer.

\section{Présences d'Antonin Artaud dans l'euvre boulézienne}

\section{Boulez et Artaud : des rencontres manquées}

Venons-en maintenant à notre seconde question préliminaire. Si Boulez envisage la musique comme "hystérie et envoûtement collectifs, violemment actuels - suivant la direction d'Antonin Artaud ", pourquoi aucune trace visible d'Artaud dans le corpus boulézien ? La réponse pourrait être tout d'abord d'ordre personnel. Selon Dominique Jameux (1985, p. 49) Boulez découvre les œuvres d'Antonin Artaud vers la fin des années 1940 grâce à Paule Thévenin 33 . Il avait rencontré cette dernière au sortir d'une conférence en privé d'Artaud avec lecture de textes encore inédits. (Boulez 2005) Cette lecture marquera le compositeur : « [...] l'avoir entendu lire ses propres textes, les accompagnant de cris, de bruits, de rythmes, nous a indiqué comment opérer une fusion du son et du mot, comment faire gicler le phonème, lorsque le mot n'en peut plus, en bref, comment organiser le délire. » (Boulez 1966, p. 62) Lui a-t-il été présenté ? Rien ne permet de l'affirmer 34 .

Musicalement, le rapprochement de Boulez et Artaud semblait appeler la réalisation d'un opéra. Il est assez intéressant de constater qu'à l'époque de ses réflexions sur Pelléas, c'est-à-dire à la fin des années 1960, le compositeur se consacre pleinement au répertoire lyrique : depuis 1966, il dirige Wagner à Bayreuth 35 , il monte Pelléas en 1969, il dirigera bientôt Wozzeck à Paris, et il commence aussi à envisager la composition d'un opus personnel. Comme Debussy, il faut reconnaître que Boulez peine à achever ses projets scéniques, et en digne héritier de la tradition joycienne du work in progress, il ne parviendra pas même à terminer un seul opéra. Nombreux furent les projets envisagés, et parfois seulement esquissés; il en est quelques-uns qui méritent ici notre attention. ${ }^{36}$ Tout d'abord son essai de collaboration avec l'écrivain Jean Genet

31 Héliogabale ou l'anarchiste couronné, paru en 1934. Tout en étant œuvre de poète, Héliogabale est surtout l'œuvre la plus documentée d'Artaud, fruit de longues et patientes recherches en bibliothèque, avec assistants. En cela, elle admet une dimension plus scientifique que les Tarahumaras, plus ethnographiques, en ce qui concerne les descriptions rituelles.

32 Et dans une grande tradition de héros d'opéras : Macbeth, Boris Godounov...

33 Assidue du Domaine Musical et membre du cénacle boulézien de la première heure, Paule Thévenin est surtout connue comme l'éditrice des Euvres Complètes d'Antonin Artaud; on lui doit aussi l'édition des premiers écrits de Boulez entre 1960 et 1961 (Relevés d'Apprenti en 1966 Le pays fertile - Paul Klee en 1989).

34 Contrairement à Henri Michaux, qui gardera d'ailleurs un souvenir plus que mitigé du concert privé des Sonates et interludes de John Cage en 1945. Sans parler de René Char avec qui il correspond.

35 De 1966 à 1970, avant la Tétralogie du centenaire en 1976.

36 Voir pour plus de renseignements Bassetto 2006. 
qui devait lui fournir un livret lié à la pègre marseillaise. ${ }^{37}$ Ce projet n'aboutira jamais et Boulez envisagera une refonte en 1966 avec trois autres sources littéraires : la Lettre du voyant de Rimbaud, Misérable Miracle, L'Infini turbulent et Paix dans les brisements de Michaux, mais aussi - et surtout - Tutuguri, Le rite $d u$ soleil noir d'Artaud. On ignore dans quelle mesure ces trois sources auraient été traitées : abandon également ${ }^{38}$ De quoi était-il question dans Tutuguri ? Pour comprendre ce rite du soleil noir, il faut pénétrer les écrits d'Artaud ainsi que leur signification profonde, tant poétique qu'ethnographique.

\section{Artaud, les Tarahumaras et Tutuguri, rite du soleil noir}

En 1936, fuyant l'Europe face à son échec pour imposer le théâtre dont il rêve ${ }^{39}$, Artaud n'a qu'un souhait : " rencontrer les indiens qui vivent dans la haute sierra tarahumara et dont les rites sont inchangés depuis des siècles. " (Thévenin 1987, p. 8) En réalité : trouver une solution à sa dépendance toxicomane à travers le rite du Ciguri, et l'absorption du Peyotl. $4^{\circ}$ C'est ce rituel qu'Artaud mettra par deux fois en poème sous le nom de Tutuguri, le rite du soleil noir. Deux versions différentes, publiées séparément dans deux ouvrages distincts ${ }^{41}$. Boulez retiendra la seconde, publiée dans les Tarahumaras ${ }^{42}$. Or ce projet de Tutuguri en $1966^{43}$ ne verra pas non plus le jour : première rencontre musicale avortée entre les deux créateurs. Définitivement ? Peut-être pas... nous y reviendrons.

Héliogabale : un second projet avorté ?44 CEuvre controversée d'Artaud, Héliogabale témoigne de l'intérêt récurrent de l'auteur du Théâtre de la cruauté pour le concept du rituel, campé cette fois-ci dans l'environnement de la Rome pourrissante du troisième siècle. Autour de la vie dépravée de l'empereur nous

37 Non à cause de la mort du dramaturge comme on le croit souvent, mais plutôt en raison du manque de compatibilité dans l'approche collaborative des deux créateurs.

38 Contrairement à Wolfgang Rihm avec son Tutuguri. Poème dansé, composé en 1981-1982. Boulez recyclera quant à lui son projet en Marges pour percussions. Également inachevé.

39 Le « théâtre de la cruauté », pour lequel il avait envisagé, dès 1933, un premier spectacle intitulé La conquête du Mexique.

40 Petit cactus consommé parfois pour ses effets psychotropes hallucinogènes; on en tire surtout la mescaline, un alcaloïde dont Henri Michaux notamment faisait usage (cf. ses recueils Misérable miracle ou Paix dans les brisements).

41 Sollicité fin 1947 pour écrire une émission pour la Radiodiffusion Française, Artaud compose Pour en finir avec le jugement de Dieu, dans lequel il insère son poème Tutuguri, le rite du soleil noir daté de 1936. L'émission ayant été interdite, le texte fut publié par K Editeur. Artaud dut donc composer un autre Tutuguri pour les Tarahumaras, ayant promis par contrat un texte intitulé le rite du soleil à Marc Barbezat. L'ouvre Pour en finir avec le jugement de Dieu existe quant à elle en document sonore (Signature, avril 2001), ainsi qu’en texte édité (collection Poésie/Gallimard, 1974).

42 « [...] Des textes d'Artaud sur les Tarahumaras, sur un rite du Peyotl. Je choisirai en particulier un texte qui est très beau et qui s'intitule Tutuguri, le rite du soleil noir. Ce livre d'Artaud est paru à l'Arbalète chez Marc Barbezat. [...] » (Boulez à Maurice Béjart, Baden-Baden, le 13 juillet 1966).

43 Dans sa lettre du 13 juillet 1966, op. cit.

44 Héliogabale ou l'anarchiste couronné, paru en 1934. Tout en étant œuvre de poète, Héliogabale est surtout l'œuvre la plus documentée d'Artaud, fruit de longues et patientes recherches en bibliothèque, avec assistants. En cela, elle admet une dimension plus scientifique que les Tarahumaras, en ce qui concerne les descriptions rituelles. Édité par Gallimard, Paris, 1979. 
trouvons bon nombre de récits de rites orientaux associés au temple d'Ẻmèse, centre religieux consacré à un dieu solaire appelé Élagabal. Mais si ce soleil n'est pas noir, il y est tout de même question d'un bétyle noir. ${ }^{45}$ En 1968, deux documents épistolaires de Jean Vilar ${ }^{46}$ adressés à Boulez font référence à ce texte. Le 10 janvier puis le 4 mars :

Parmi les milliers de choses dont nous avons à nous entretenir, j'ai oublié de vous faire part de la demande de Béjart lors de notre dernière entrevue. Il m’a prié de vous rappeler le projet « Héliogabale » d'après l'œuvre d'Antonin Artaud. [...]

J'ai d'autre part rencontré Béjart : une liste d'œuvres a été dressée dont je vous parlerai à notre prochaine entrevue. Entre autres choses il compte sur votre Héliogabale. (cité dans Bassetto 2006, p. 267)

Hélas, aucune autre source relative au projet « Héliogabale » n’a pu être trouvée. Probablement un ballet, si Béjart en est l'instigateur. Quoi qu'il en soit, Boulez ne retentera jamais plus l'aventure scénique après ce second échec de rapprochement artistique avec Artaud. En revanche, il va se tourner vers un autre concept, le plus cher à Antonin Artaud : le Rituel.

\section{Rituel, in memoriam Bruno Maderna}

Boulez s'est toujours intéressé aux rituels et particulièrement dans les cultures africaines et sud-américaines. Par exemple, voici ce qu'écrivait Schaeffner au compositeur le 26 décembre 1961 :

Je vous enverrai prochainement des tirés à part de deux articles de moi que vous ne connaissez certainement pas; ils sont plus ethnographiques que musicologiques; l'un est une étude complète d'un rituel africain, l'autre pose des problèmes au sujet de la participation des musiciens à des rituels (également africains). (Boulez et Schaeffner 1998, p. 58)

Cette réponse fait suite à une demande de Boulez concernant des instruments africains dont il aimerait faire usage :

Autre sujet, et dernier de cette lettre - du moins je l'espère ! - : les tambours de bois ! Je suis prêt à tous les « sacrifices » pour ce facteur, et à tous les «cadeaux ", s'il consent à laisser un instrument entre les mains d'un mécréant. Mais je me promets bien du plaisir à lire sa lettre, en attendant celui de jouer sur les tambours! Pour l'instant, toutefois, la pirogue se révèle encore inutile. Mais je ne désespère pas un jour, $d$ ' " embarquer » les musiciens, sinon la musique, sur un pareil esquif !! » (Boulez et Schaeffner 1998, p. 35)

Boulez approche lui-même différents rituels durant ses propres voyages : lors du second déplacement de la Compagnie Madeleine Renaud et Jean-Louis

45 Une pierre noire conique tombée du ciel.

46 Vilar, Béjart et Boulez travaillent alors à l'élaboration du fameux projet de réforme de l'opéra, malgré la brouille du compositeur avec André Malraux et Marcel Landowski. 
Barrault au Brésil en juillet 1954, il assiste à des rites de " macumba » dans l'arrière pays de Salvador (Bahia). Cette expérience suggérera à Jean-Louis Barrault la mise en scène de l'Orestie d'Eschyle, pour laquelle Boulez écrira sa fameuse musique de scène éponyme ${ }^{47}$. Mais il existe une création plus intéressante : de 1970 à 1974 Boulez compose Rituel ${ }^{48}$, une ouvre possédant un degré d'ouverture, spatialisée (donc également en lien avec les réflexions scéniques de Boulez) et faisant un usage particulier des percussions résonnantes, celles-là même évoquées par Debussy dans les rites annamites. L'insertion de ces instruments spécifiques n'est pas nouvelle chez Boulez, elles apparaissaient dans la neuvième et dernière pièce du Marteau sans maître (seconde version). 49 $\mathrm{Si}$ aujourd'hui ces percussions font partie de l'instrumentarium standard des orchestres, l'accès à ces métaux, notamment pour la musique de chambre, n'était pas chose aisée en 1955. C’est une fois encore Schaeffner qui rend service à Boulez :

Je dois redonner à Paris pour la première fois depuis deux ans, le Marteau sans maître dans le nouveau bâtiment de l'Unesco, le 28 octobre $^{50}$. Je voulais vous demander s'il était encore possible d'emprunter chez vous, comme en 1956, les magnifiques gongs et tam-tams qui sont si utiles à la bonne fin de ce Marteau, qui - sans cela - risque d'être fort bancal ${ }^{51}$. Pourrions nous encore passer en catimini par les caves et vous emprunter cela l'espace de 5 ou 6 jours?

À l'époque des conversations sur Pelléas, Boulez règlera cet écueil :

[...] Je viens, par ailleurs, de recevoir (occasion de Hambourg) douze gongs thailandais à mamelon, de toute beauté; et pour un prix relativement modéré, ce qui n'a rien gâté de leur beauté; si vous venez dans ces parages, je vous les ferai entendre ${ }^{22}$ ! (Boulez et Schaeffner 1998, p. 35-36)

Dans sa version actuelle (et a priori définitive), Rituel est probablement l'une des compositions les plus rigoureuses de son auteur : entièrement fondée sur le chiffre 7 et sur des permutations à sept valeurs, auxquelles sont soumis tous les paramètres. L'œuvre se structure en 15 sections : 14 premières alternant deux types de séquences contrastées (paires et impaires), et une quinzième équivalente à la somme des 14 précédentes, c'est-à-dire un grand mouvement arsis/ thésis (tension/détente) parfaitement équilibré. Huit groupes instrumentaux

47 Une page des esquisses relatives à la musique de scène de l'Orestie conservées à la Fondation Paul Sacher porte l'indication " comme la macumba ». Il existe plusieurs témoignages de l'intérêt des deux artistes pour ce rite. Voir Valère et Dessailly 1996, p. 126, Barrault 1955 p. $94-95$ et p. 100 et Boulez, [juin 1994] Communication personnelle à Rosângela Pereira de Tugny (Boulez et Schaeffner 1998, p. 52-53).

48 Qui deviendra par la suite Rituel in memoriam Bruno Maderna.

49 Création partielle à Baden-Baden le 18 juin 1955 sous la direction de Hans Rosbaud, et donné en création française au Petit Théâtre de Marigny en mars 1956, sous la direction du compositeur.

50 Ce concert (28 octobre 1958) était organisé sur invitation. Au programme : musique d'Inde par Ravi Shankar et Chatur Lal, Improvisation sur Mallarmé (I et II) et le Marteau sans maître, dirigés par le compositeur.

51 Ils appartenaient au département d'ethnomusicologie du musée de l'Homme.

52 Pierre Boulez à André Schaeffner, le 21 novembre 1961. 
forment l'orchestre, ou plutôt $7+1: 7$ groupes constitués de 1 à 7 instruments (bois et cordes) associés chacun à une percussion non résonnante. Le huitième groupe, excentré, est un ensemble de 14 cuivres $(2 \times 7)$ lié à un groupe de 7 tamtams et de 7 gongs. 7 gestes musicaux ${ }^{53}$ constituent le matériau musical. Dans le discours, on constate l'accumulation des groupes de 1 à 7 dans les sections paires, 1 à 7 notes dans les anacrouses et désinences, etc. Cette rigueur se retrouve dans l'une des esquisses de Rituel : une simple page envoyée par le compositeur et intitulée Sept interjections à Pierre Souvtchinski. ${ }^{54}$ Sur cette page : 7 séquences musicales (qui deviendront les 7 gestes fondamentaux de Rituel) et quelques explications griffonnées. Accompagnant cette page : deux lettres non datées envoyées de Londres et de New York par Boulez à Souvtchinski. En les lisant simultanément, ces deux lettres prennent tout leur sens. Le compositeur y fait référence à une magie du chiffre 7 , magie que nous retrouvons dans Rituel, de même que la forme en quatorze points alternés $(2 \times 7)$ des sections I à XIV. Nous les reproduisons ici en regard:

Cher Pierre,
1. De Londres à New York
1. et de New York à Londres
2. ma pensée
2. n'oublie pas
3. l'ami de toujours.
3. La circonstance
4. exceptionnelle
4. me le fait souligner.
5. Ces interjections
5. sont sept
6. chiffre en principe magique.
6. cette magie
7. je la désire propice
7. pour vous
cher Pierre.

Oserions-nous une corrélation avec l'œuvre d'Artaud ? Ce dernier fait constamment référence à la magie des nombres. Par exemple dans l'une de ses nombreuses lettres de Rodez au docteur Ferdière, datée du 18 octobre 1943 : " [...] Mais ce Rien du tout il y a mis des chiffres et des Nombres $3-7$ avec 10 - et jusqu'à 12 le chiffre de la maturité dans les formes et il a jeté au milieu le signe de la croix. » (Artaud 1977, p. 71) Dans Tutuguri, rite du soleil noir, il va même plus loin en intégrant cette magie des nombres et ce signe de la croix au rite du Peyotl des Tarahumaras :
[...]
Et il y a six hommes
un pour chaque soleil
et un septième homme
qui est le soleil tout
$\mathrm{cru}$
habillé de noir et de chair rouge.

Le Rite est que le nouveau soleil passe par sept

points avant d'éclater à l'orifice de la terre.

53 Issus des encadrés de la matrice originelle d'...explosante-fixe... de 1972.

54 Datée du 19 octobre 1972 et conservée à la Bibliothèque Nationale de France, section musique, Ms. 21611. 


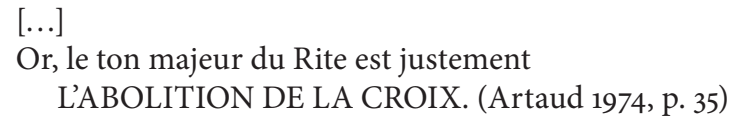

Artaud lie et spatialise magie du chiffre 7 et signe de la croix; Boulez quant à lui associe cette magie aux jeux de spatialisation et d'accumulation des différents groupes instrumentaux. Plus que probablement le compositeur se serat-il remémoré son intérêt (1966) pour ce texte. Mais si l'on veut pousser encore plus loin la comparaison entre Rituel et l'œuvre d'Artaud, un autre passage de Tutuguri mérite toute notre attention :

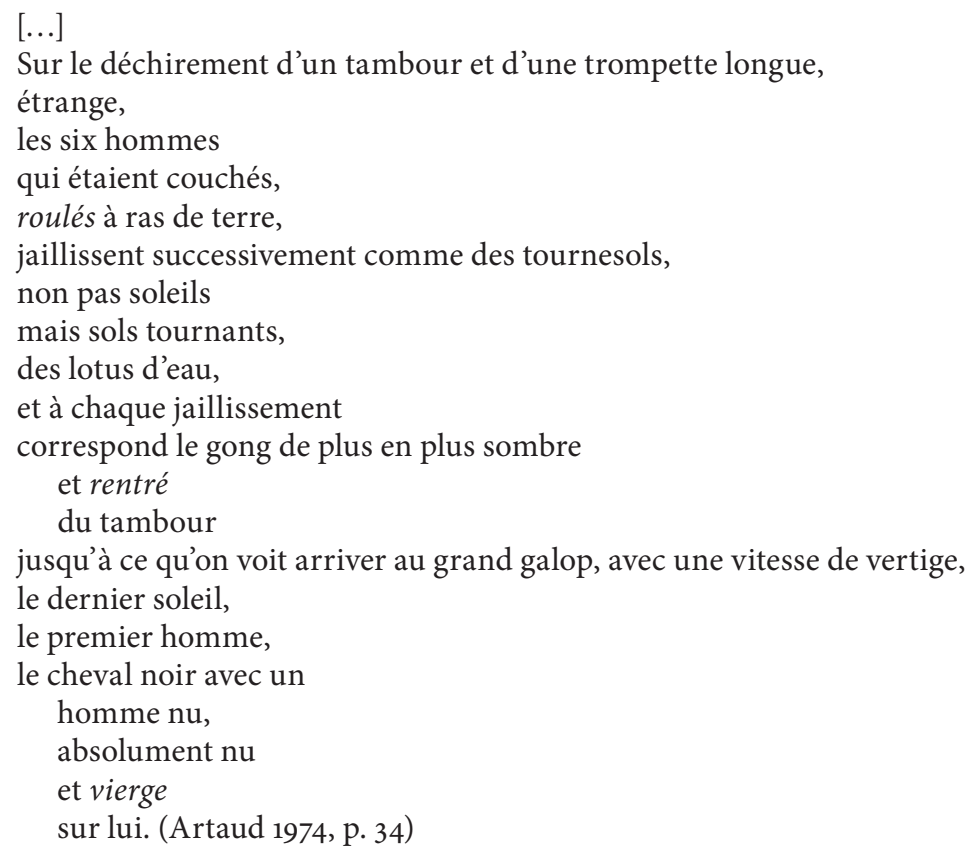

Difficile de résister à la tentation de rapprocher ce passage des premières sections de Rituel : jaillissement croissant et régulier des groupes instrumentaux (de 1 à 8 des sections I à XIV). De même sont frappantes les indications instrumentales du poème : "le déchirement d'un tambour et d'une trompette longue » évoque irrémédiablement le solo de la houtbois accompagnée de son tabla de la section II; ou encore les gongs ponctuant les jaillissements des six hommes, tels les tam-tams de Boulez annonçant les interjections des groupes dans les sections impaires. Signalons enfin que ces associations instrumentales en lien avec le concept du rituel sont fréquentes chez Artaud. Par exemple dans la prose d'Héliogabale:

Le culte de Baal à Emèse représenté par la vigoureuse verge d’Ẻlagabale, dieu noir, faisait pendant, par ses rites complexes et surchargés, au culte de Tanit Astarté, la lune, qui, à quelques kilomètres de là, sévissait dans les profondeurs fraîches du temple d'Hiérapolis. [...] Au moment de l'apparition du dieu, du dieu ivre qui fait tituber ses gardes, le temple vibre, 
en harmonie avec les tourbillons stratifiés des sous-sols, connus et repérés depuis la plus haute antiquité. Dans les chambres des rites, et jusqu'à plusieurs centaines de mètres au dessous du sol, les veilleurs se passent le mot, donnent de la voix, heurtent des gongs, font gémir des trompes dont les voûtes se renvoient les échos. (Artaud 1979, p. 30-31)

\section{Conclusion}

Ainsi, il semble opportun de réévaluer l'ascendant d'Antonin Artaud et son œuvre sur Boulez, dont Rituel offre un unique témoignage tangible dans l'œuvre boulézienne, pourtant passé inaperçu, mais dont l'influence souterraine s'exerce également sur la pensée et l'esthétique du compositeur. Les réflexions sur Debussy nous révèlent des idées et des concepts issus du Théâtre de la cruauté, et une théorie particulière d'interprétation, étrangers à toute orthodoxie d'école (Artaud a-t-il seulement eu une école ou des descendants, si ce n'est quelques émules disparates, et un ou deux épigones ?). L'impact d'Artaud sur Boulez transcende l'art scénique et l'interprétation et se manifeste dans l'appréhension de la «substantifique moelle » de la création, antérieure à toute actualisation dans sa mise en œuvre formelle. En toute logique, cette présence artaldienne se retrouve donc au stade "poïétique " de l'œuvre boulézienne sans transparaître au niveau esthésique (tout comme elle point dans sa correspondance privée sans émerger dans ses écrits publiés). Concluons par un détail amusant : bien que Rituel soit une œuvre achevée, Boulez ne semblait pas vouloir en rester là... Si l'on en croit le catalogue de ses esquisses 55 , il avait envisagé en 1975-1976 un nouveau Rituel, cette fois sous-titré Grand rituel! Il y renoncera. Encore. Il n'en subsiste désormais que quelques pages d'esquisses et une première ébauche. Rituel in memoriam Bruno Maderna restera donc l'incarnation la plus aboutie de la démarche rituelle de Boulez, et donc en une certaine mesure son hommage le plus éloquent à la poétique artaldienne.

\section{RÉFÉRENCES}

Artaud, Antonin. 1925. L'ombilic des Limbes. Paris : Gallimard.

—_. 1938. Le théâtre et son double. Paris : Gallimard.

. 1962. "Préface aux douze chansons de Maurice Maeterlinck ». Dans Maurice Maeterlinck, présenté par Roger Bodart. Paris : Éditions Pierre Seghers, p. 89-93. (Publ. orig. 1923.)

—_ 1974. Pour en finir avec le jugement de Dieu. Paris : Gallimard.

—_. 1977. Nouveaux écrits de Rodez. Paris : Gallimard.

—_ 1979. Héliogabale ou l'anarchiste couronné. Paris : Gallimard. (Publ. orig. Paris : Denoël \& Steele, 1934.)

—_ 2001. Van Gogh le suicidé de la société, présenté par Evelyne Grossman.

Paris : Gallimard. (Publ. orig. Paris : K, 1947.)

$552^{\mathrm{e}}$ édition établie en 2007 par Robert Piencikowski. 
_. 2003. "Le théâtre de la cruauté ». Dans Pour en finir avec le jugement de Dieu, présentée par Evelyne Grossman. Paris : Gallimard, p. 63-80. (Publ. orig. Paris : K, 1932.)

Barrault, Jean-Louis. 1955. "Eschyle et l'Orestie ", Cahiers de la Compagnie Madeleine Renaud et Jean-Louis Barrault, ${ }^{\circ}$ 11. Paris : Julliard, p. 2-118.

Bassetto, Luisa. 2006. "Marginalia, ou l'opéra-fantôme de Pierre Boulez ».

Pierre Boulez, Techniques d'écriture et enjeux esthétiques, sous la dir. de Jean-Louis Leleu et Pascal Decroupet. Genève : Contrechamps, p. 255-298. Boulez, Pierre. 1948. «Propositions ». Polyphonie n², p. 65-72; repris dans 1995.

Points de repère I : Imaginer. Paris : Christian Bourgois, p. 253-262. - 1966. «Son et verbe» (1958). Relevés l’apprenti. Paris : Seuil.

__. 1985. "Claude Debussy ». Points de repère. Paris : Christian Bourgois, p. 210-217.

_ 1985a. "Miroirs pour Pelléas et Mélisande. ». Points de repère. Paris : Christian Bourgois, p. 433-446.

—_ 1985b. Points de repère. Paris : Christian Bourgois.

_ _ 2005. «Réformer la loi sur les ayants droit». Le Nouvel Observateur, 13 Mai.

Boulez, Pierre et James Briscoe. 1999. "Debussy, Pelléas, and Orchestra Conducting: An Interview with Pierre Boulez. ». Debussy in Performance, sous la dir. de James Bricoe. New Haven et Londres : Yale University Press, p. 176-19o.

Debussy, Claude. 1927. Lettres de Claude Debussy à son éditeur. Sous la dir. de J. Durand. Paris : Durand.

Denis, Maurice. 1932. Henry Lerolle et ses amis. Paris : Durand.

Derrida, Jacques. 1967. «La parole soufflée ». Dans L'écriture et la différence. Paris : Seuil, p. 253-292. (Publ. orig. 1965 dans Tel quel no 20, p. 41-67.)

_- 1967a. «Le théâtre de la cruauté et la clôture de la représentation ». Dans L'écriture et la différence. Paris : Seuil, p. 341-368. (Publ. orig. dans Critique $\mathrm{n}^{\circ}$ 230, juillet 1966.)

Feneyrou, Laurent. 2005. "Debussy au miroir des écrits de Boulez ». Conférence donnée le 4 mars 2005 à l'ENS à Paris, inédite.

Halbreich, Harry et Edward Lockspeiser. 1989. Debussy, sa vie et sa pensée. Paris : Fayard.

Humbertclaude, Éric. 1999. La transcription dans Boulez et Murail. Paris : L'Harmattan.

Jameux, Dominique. 1985. Pierre Boulez. Paris : Fayard.

Leleu, Jean-Louis et Pascal Decroupet. 2006. Pierre Boulez, Techniques d'écriture et enjeux esthétiques. Genève: Contrechamps.

Pereira de Tugny, Rosângela. 1998. Correspondance 1954-1970. Paris : Fayard.

_. 2006. "Lautre moitié de l'art ». Pierre Boulez: Techniques d'écriture et enjeux esthétiques, sous la dir. Jean-Louis Leleu et Pascal Decroupet. Genève : Contrechamps, p. 299-317.

Prevel, Jacques. 1994. En compagnie d'Antonin Artaud, suivi de Poèmes. Paris : Flammarion. 
Schaeffner, André. 1980. «Variations Schoenberg ». Dans Essais de musicologie et autres fantaisies. Paris : Le Sycomore. (Publ. orig. dans Contrepoints, $\mathrm{n}^{\circ} 7$, 1951.)

. 1998. Variations sur la musique. Paris : Fayard.

Schaeffner, André et Pierre Boulez. 1998. Correspondance 1954-1970, présentée et annotée par Rosângela de Tugny. Paris : Fayard.

Thévenin, Paule. 1987. «Préface ». Tarahumaras. Paris : Gallimard.

Valère, Simone et Jean Dessailly. 1996. Un destin pour deux. Paris : Ramsay.

\title{
RÉSUMÉ
}

Dans un texte de 1948, Pierre Boulez exprime son aspiration à investir sa musique d'un souffle rituel comparable à celui de la poétique d'Antonin Artaud. Ces propos soulèvent de nombreuses questions quant à l'influence artaldienne, touchant à la fois les activités de chef d'orchestre de Boulez et la conception de ses œuvres. Cet article s'attarde d’abord aux considérations de Boulez sur Pelléas et Mélisande dans ses écrits publics, et aux liens qu'il établit entre Debussy et Artaud dans ses écrits privés. Une seconde partie se concentre sur les liens sous-jacents qui relient l'œuvre d'Artaud et celle de Boulez, plus particulièrement dans Rituel, in memoriam Bruno Maderna.

\begin{abstract}
In a 1948 text, Pierre Boulez expresses his desire to infuse his music with a ritualistic undertone comparable to that of Antonin Artaud's poetic. This remark raises questions about Artaud's influence, concerning both Boulez's conducting activities and the conception of his works. The first part of this article deals with Boulez's public writings on Pelléas et Mélisande and on the links he establishes between Debussy and Artaud in his private writings. The second part concentrates on the connections that relate Artaud's works to those of Boulez, particularly in Rituel, in memoriam Bruno Maderna.
\end{abstract}

